\title{
Searching for Galactic Hidden Gas through interstellar scintillation: The OSER project
}

\section{Moniez}

Laboratoire de l'Accélérateur Linéaire, IN2P3 CNRS, Université Paris-Sud, 91405 Orsay

Cedex, France

E-mail: moniezalal.in2p3.fr

\section{R. Ansari}

Laboratoire de l'Accélérateur Linéaire, IN2P3 CNRS, Université Paris-Sud, 91405 Orsay

Cedex, France

E-mail: ansarialal.in2p3.fr

\section{F. Habibi}

Laboratoire de l'Accélérateur Linéaire, IN2P3 CNRS, Université Paris-Sud, 91405 Orsay Cedex, France

Department of Physics, Sharif University of Technology PO Box 11365-9161, Tehran, Iran E-mail: habibielal.in2p3.fr

Considering the results of baryonic compact massive objects searches through microlensing [1], cool molecular hydrogen $\left(\mathrm{H}_{2}-\mathrm{He}\right)$ clouds should now be seriously considered as a possible major component of the Galactic hidden matter. It has been suggested that a hierarchical structure of cold $\mathrm{H}_{2}$ could fill the Galactic thick disk [8] or halo [3], providing a solution for the Galactic hidden matter problem. This gas should form transparent "clumpuscules" of $\sim 10$ AU size, with a column density of $10^{24-25} \mathrm{~cm}^{-2}$, and a surface filling factor smaller than $1 \%$. The OSER project (Optical Scintillation by Extraterrestrial Refractors) is proposed to search for scintillation of extragalactic sources through these Galactic - disk or halo - transparent $\mathrm{H}_{2}$ clouds. This project should allow one to detect (stochastic) transverse gradients of column density in cool Galactic molecular clouds of order of $\sim 3 \times 10^{-5} \mathrm{~g} / \mathrm{cm}^{2} / 10000 \mathrm{~km}$.

We present the expectations from a complete simulation of the interstellar scintillation process. Test observations have been obtained through known nebulae to probe the concept in a controlled situation; we show preliminary results from their analysis.

Identification of dark matter 2008

August 18-22, 2008

Stockholm, Sweden

\footnotetext{
* Speaker.
} 


\section{Introduction: The signature of transparent $\mathrm{H}_{2}-\mathrm{He}$ inhomogeneous clouds}

Refraction through an inhomogeneous transparent cloud (hereafter called screen) distorts the wave-front of incident electromagnetic waves (Fig.1)[6]. The amplitude in the observer's plane after propagation is described by the Huygens-Fresnel diffraction theory. For a point-like source, the intensity in the observer's plane is affected by interferences which, in the case of stochastic inhomogeneities, takes on the speckle aspect. At least 3 distance scales characterize this speckle:

- The diffusion radius $R_{\text {diff }}$ of the screen, defined as the transverse separation for which the root mean square of the phase difference is 1 radian [7]. $R_{\text {diff }}$ characterizes the cloud turbulence.

- The Fresnel radius

$$
R_{F}=\sqrt{\frac{\lambda z_{0}}{2 \pi}} \sim 2221 \mathrm{~km}\left[\frac{\lambda}{1 \mu \mathrm{m}}\right]^{\frac{1}{2}}\left[\frac{z_{0}}{1 \mathrm{kpc}}\right]^{\frac{1}{2}},
$$

of order of $1000 \mathrm{~km}$ to $7000 \mathrm{~km}$ at $\lambda=1000 \mathrm{~nm}$, for a screen distance $z_{0}$ between $125 \mathrm{pc}$ (distance of the Bok globule B68) to $10 \mathrm{kpc}$ (typical distance of a halo-clumpuscule). The Fresnel radius characterizes the diffraction process.

- The refraction radius

$$
R_{\text {ref }}=\frac{2 \pi R_{F}^{2}}{R_{\text {diff }}}=\frac{\lambda z_{0}}{R \text { diff }} \sim 31,000 \mathrm{~km}\left[\frac{\lambda}{1 \mu \mathrm{m}}\right]\left[\frac{z_{0}}{1 \mathrm{kpc}}\right]\left[\frac{R_{\text {diff }}}{1000 \mathrm{~km}}\right]^{-1},
$$

size of the diffraction image in the observer's plane from a patch of $R_{\text {diff }}$ in the screens plane. In addition, long scale structure of the screen can induce local focusing/defocusing configurations that produce intensity variations.

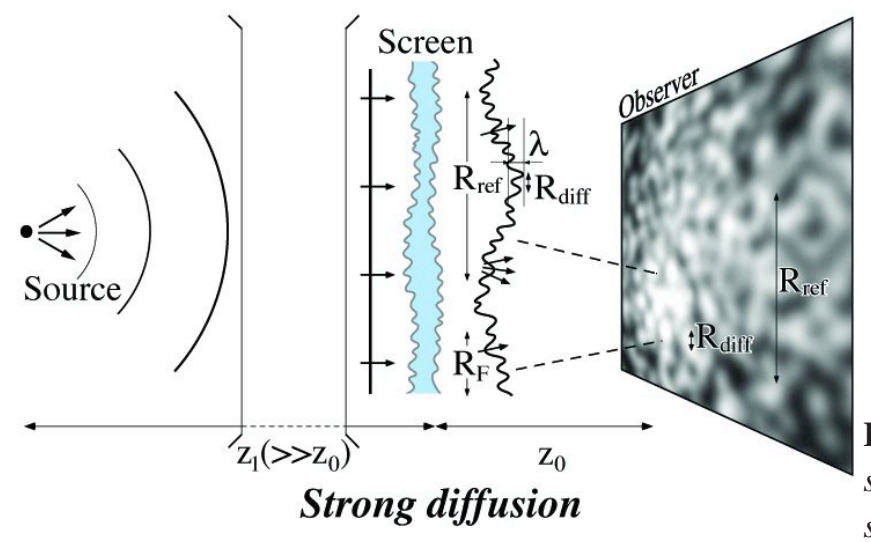

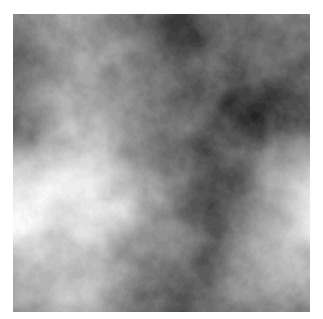

Figure 1: Illumination pattern from a point source (left) after crossing a stochastic phase screen (simulation with levels of grey)(up).

\section{Expectations from simulation: intensity modulation, time scale, color effects}

After crossing an inhomogeneous cloud described by the Kolmogorov turbulence law (Fig.1, right), the light from a monochromatic point source produces an illumination pattern on Earth made of speckles of size $R_{\text {diff } f}$ within larger structures of size $R_{\text {ref }}$ (see Fig.3, up-left). The illumination pattern from a stellar source of radius $r_{s}$ is the convolution of the point-like intensity pattern with the projected intensity profile of the source (projected radius $R_{S}=r_{s} \times z_{0} / z_{1}$ ) (Fig.3, up-center). In 
general, the small speckle is almost completely smoothed, and only the large structures of size $R_{r e f}$ shows a significant modulation, that decreases when the ratio

$$
\frac{R_{S}}{R_{\text {ref }}}=\frac{r_{s} R_{\text {diff }}}{\lambda z_{1}} \sim 2.25\left[\frac{r_{s}}{R_{\odot}}\right]\left[\frac{R_{\text {diff }}}{1000 \mathrm{~km}}\right]\left[\frac{\lambda}{1 \mu \mathrm{m}}\right]^{-1}\left[\frac{z_{1}}{10 \mathrm{kpc}}\right]^{-1}
$$

increases (Fig.2). For a cloud moving with a transverse velocity $V_{T}$ relative to the line of sight, this

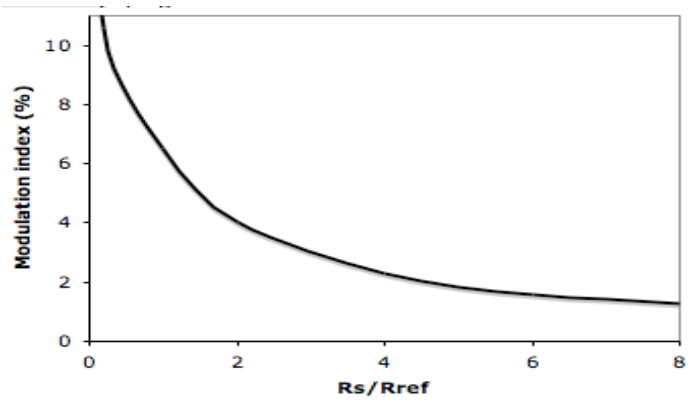

Figure 2: Intensity modulation index $m=\sigma_{I} / \bar{I}$ of a stellar light-curve as a function of $R_{S} / R_{\text {ref }}$.

pattern sweeps across the Earth at the same speed, inducing intensity fluctuations with characteristic time scale

$$
t_{\text {ref }}(\lambda)=\frac{R_{\text {ref }}}{V_{T}} \sim 5.2 \text { minutes }\left[\frac{\lambda}{1 \mu m}\right]\left[\frac{z_{0}}{1 \mathrm{kpc}}\right]\left[\frac{R_{\text {diff }}}{1000 \mathrm{~km}}\right]^{-1}\left[\frac{V_{T}}{100 \mathrm{~km} / \mathrm{s}}\right]^{-1} .
$$

We will assume that the scintillation is mainly due to this screen motion rather than to the pattern variation due to the cloud structure evolution, as it is the case in radioastronomy observations [5] (frozen screen approximation).

For the Galactic $\mathrm{H}_{2}-\mathrm{He}$ clouds we are interested in, $R_{S} / R_{\text {ref }} \sim\left(r_{s} / R_{\odot}\right) \times\left(R_{\text {diff }} / 1200 \mathrm{~km}\right)$ at $\lambda=500 \mathrm{~nm}$, assuming that the scintillating star is in the Large Magellanic Cloud (LMC). Therefore, at optical wavelength, one expects a relatively high intensity modulation index $\left(m=\sigma_{I} / \bar{I}>1 \%\right)$ at time scale $t_{\text {ref }} \gtrsim 2$ minutes (if $V_{T}=200 \mathrm{~km} / \mathrm{s}$ ) for ordinary LMC stars (of radius $R_{S}=R_{\odot}$ ) as soon as $R_{\text {diff }} \lesssim 6500 \mathrm{~km}$. As $R_{\text {ref }}$ depends on $\lambda$, one expects a significant variation of the characteristic time scale $t_{\text {ref }}(\lambda)$ between the red side of the optical spectrum and the blue side. This property is probably the best signature of the scintillation as it indicates a propagation effect, incompatible with any type of intrinsic source variability.

\section{Toward an experimental setup for searching hidden matter}

The search for scintillation induced by transparent Galactic molecular clouds needs the capability to sample at the sub-minute scale the luminosity of LMC or SMC main sequence stars with a photometric precision better than $1 \%$. This performance can be achieved using a two meter class telescope, with a high quantum efficiency detector and a short dead-time between exposures. Multi-wavelength detection capability is necessary to exploit the dependence of the characteristic time scale with the wavelength.

As mentioned in [8], gas clumpuscules would cover $1 \%$ of the sky surface if they fill the halo. Therefore, the probability for a star to be subject to a scintillation with a modulation index $m>m_{\text {threshold }}$ is $\tau_{\text {scint }}=\alpha \times 1 \% \times \beta\left(m_{\text {threshold }}\right)$, where $\alpha$ is the fraction of halo made of gaseous objects and $\beta$ ( $\left.m_{\text {threshold }}\right)$ is the probability for the cloud to produce scintillation with $m>m_{\text {threshold }}$. 

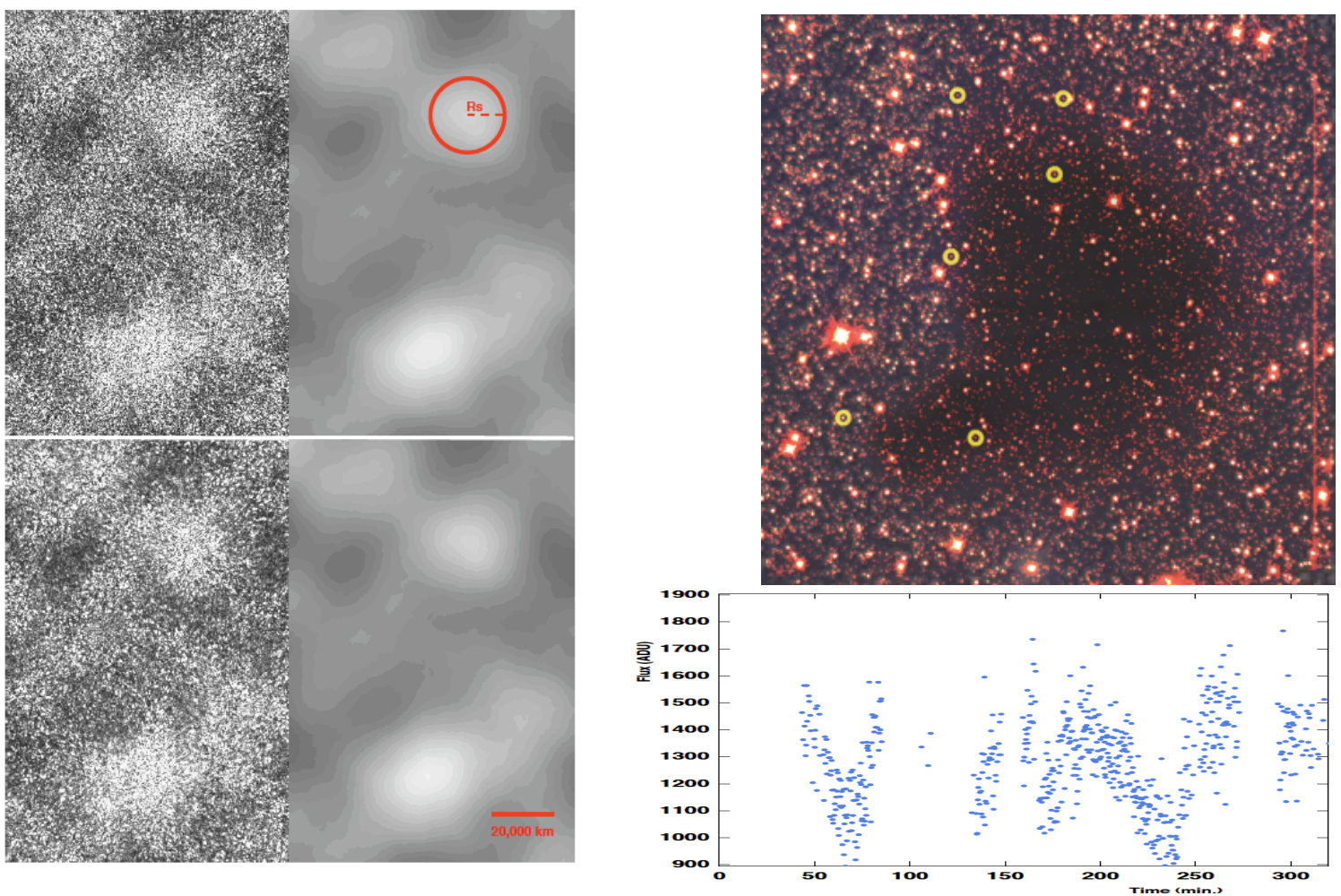

Figure 3: Left panels: Illumination map at $\lambda=2.18 \mu$ on Earth from a point source (up-left)- and from a $K O V \operatorname{star}\left(r_{s}=0.85 R_{\odot}, M_{V}=5.9\right)$ at $8 \mathrm{kpc}$ (right). The bottom maps are illuminations in $K_{s}$ wide band. The refracting cloud is assumed to be at $160 \mathrm{pc}$. The red scale represents 20,000 km. Right panels: Position of the 6 most variable stars on a combined visible+IR image showing the cloud geometry (up) and one of their light-curves (down).

As $m$ is related to $R_{\text {diff }}$ and to the source distance and radius, the $\beta$ function depends only on the $R_{\text {diff }}$ distribution when the source is known. Considering the lack of knowledge on the clouds turbulence, and the exploratory aspect of this program, we need a wide field detector to monitor many main-sequence small stars.

Foreground effects, background to the signal: Conveniently, atmospheric intensity scintillation is negligible through a large telescope $\left(m_{\text {scint }} \ll 1 \%\right.$ for a $>1 \mathrm{~m}$ diameter telescope [4]). Any other atmospheric effect such as absorption variations at the minute scale (due to fast cirruses for example) should be easy to recognize as long as nearby stars are monitored together. Asterosismology, granularity of the stellar surface, spots or eruptions produce variations of very different amplitudes and time scales. A rare type of recurrent variable stars exhibit emission variations at the minute scale, but they could be identified from their spectrum.

\section{Preliminary studies with the NTT}

To test the concept, we searched for gas induced scintillation of stars located behind visible nebulae with the ESO-NTT. In two nights of June 2006 we got 4749 consecutive exposures of $T_{\exp }=10 \mathrm{~s}$, taken with the infra-red SOFI detector in $K_{s}$ and $J$ towards B68, cb131, Circinus and 
SMC. For a future search for transparent hidden matter, visible light - corresponding to the maximum stellar emission - would be more optimal. Through the cores of B68, cb131 and Circinus, gas column densities of $\sim 10^{22}$ atoms $/ \mathrm{cm}^{2}$ induce an average phase delay of $250 \times 2 \pi$ at $\lambda=2.18 \mu$. According to our studies, a few percent scintillation signal is expected from stars smaller than the sun, if relative column density fluctuations of only $\sim 10^{-3}$ occur within less than a few thousand kilometers (corresponding to $R_{\text {diff }} \lesssim 2000 \mathrm{~km}$ ). Such small relative fluctuations cannot induce significant absorption variations, and we will neglect them. The expected time scale of the scintillation is $t_{\text {ref }} \gtrsim 5$ minutes, assuming $V_{T} \sim 20 \mathrm{~km} / \mathrm{s}$. We produced the light curves of 9599 stars with $\sim 2000$ measurements, using the EROS software [2]. We searched for stochastic luminosity fluctuations on the 788 best measured stars (i.e. with photometric precision better than 10\%). About 1/4 of them are located behind the cloud. After blind selection and identification of known artifacts (hot pixels, dead zones, bright egrets...), we found only 6 stars with significant variability (Fig.3, down-right), all of six interestingly located near the Bok globule limit and none outside the globule (Fig.3, up-right). Their status should be clarified with the analysis of their light curve time structure (to be done), but the confirmation of a scintillation signal would probably need complementary observations. Nevertheless we can already conclude that the signal we are searching for should not be overwhelmed by background. The next steps should be to further investigate the correlation between the variability and the estimated gas column density, and to study the chromaticity of the intensity fluctuations, that need simultaneous observations with different filters.

\section{Conclusions and perspectives}

The opportunity to search for scintillation results from the subtle coincidence between the arm-lever of interference patterns due to hypothetic turbulent gas in the Milky-Way and the angular size of the stars. The hardware and software techniques required for such searches are available just now, and a light project could be operational within a few years. Alternatives under study are the use of the data from the LSST project and from the GAIA mission. If a scintillation indication is found, one will have to consider an ambitious project involving synchronized telescopes, a few thousand kilometers apart. Such a project would allow to temporally and spatially sample an interference pattern, unambiguously providing the refractive length scale $R_{r e f}$, the speed and the dynamics of the scattering medium.

\section{References}

[1] Afonso, C., et al. (EROS coll.), A\&A 400, 951 (2003).

[2] Ansari, R., 1996, Vistas in Astronomy, 40, No 4

[3] De Paolis, F. et al., PRL 74, 14 (1995)

[4] Dravins, D. et al., Pub. of the Ast. Soc. of the Pacific 109 (I, II) (1997), 110 (III) (1998).

[5] Lyne, A.G. \& F. Graham-Smith, Pulsar Astronomy, Cambridge University Press (1998)

[6] Moniez, M., A\&A 412, 105 (2003).

[7] Narayan, R. Phil. Trans. R. Soc. Lond. A 341, 151 (1992)

[8] Pfenniger, D. \& Combes, F., A\&A 285, 94 (1994). 